\title{
LETTER Hybrid Integration of Visual Attention Model into Image Quality Metric
}

\author{
Chanho JUNG ${ }^{\dagger a)}$, Member
}

\begin{abstract}
SUMMARY Integrating the visual attention (VA) model into an objective image quality metric is a rapidly evolving area in modern image quality assessment (IQA) research due to the significant opportunities the VA information presents. So far, in the literature, it has been suggested to use either a task-free saliency map or a quality-task one for the integration into quality metric. A hybrid integration approach which takes the advantages of both saliency maps is presented in this paper. We compare our hybrid integration scheme with existing integration schemes using simple quality metrics. Results show that the proposed method performs better than the previous techniques in terms of prediction accuracy.

key words: image quality metric, visual attention (VA), hybrid integration
\end{abstract}

\section{Introduction}

Machine evaluation of image quality plays an essential role in a wide range of applications such as image acquisition, processing, compression, transmission, reproduction, display, and so on [1]. For this reason, developing objective image quality assessment (IQA) methods has attracted much research interest over the past decade. In the literature, a large number of image quality metrics have been proposed. Depending upon the availability of reference image, they are mainly classified as full-reference (FR), reduced-reference (RR), and no-reference (NR) metrics. Each of these three types of metrics has its own usefulness and applications, and this paper gives particular attention to the problem of "FR" IQA.

Many researchers have devoted their efforts to incorporate relevant characteristics of the human visual system into image quality measures. This is because the ultimate goal of IQA research is to predict the image quality as perceived by human subjects. In recent years, integration of visual attention (VA) model into image quality metric has emerged as a promising solution [2]-[5]. Such an approach is based on the assumption that distortions occurring in visually salient areas might be more visible, and thus more annoying [3], [5]. Quantitative methods of predicting VA are mainly classified into two categories: one is based on eye-tracking equipment, while the other on computational VA models. The former provides the ground truth VA data (i.e., subjective saliency map), but the deployment of such systems is

\footnotetext{
Manuscript received July 11, 2014
}

Manuscript revised August 11, 2014.

Manuscript publicized August 22, 2014

†The author is with the IT Convergence Technology Research Laboratory, Electronics and Telecommunications Research Institute (ETRI), Yuseong-Gu, Daejeon 305-700, Republic of Korea.

a) E-mail: peterjung@etri.re.kr (Corresponding author)

DOI: 10.1587/transinf.2014EDL8141 usually unrealistic for real-time applications [3], [4]. On the other hand, the latter has the advantage that it enables automated deployment in various image processing systems [4], [5]. Due to this reason, numerous methods which integrate the computational VA model into quality measures continue to be reported [2], [4], [6], [7], and we focus on such a potential paradigm in this paper. The fundamental idea behind the integration is to weight image quality measurements by objective saliency map obtained from the computational VA model. Existing methods use either a "task-free" saliency map for an original image or a "quality-task" one for a test image in the design of VA-based objective metrics. Some researchers have compared the benefits of the two policies [4], [5]. In this paper, we propose a hybrid integration approach which simultaneously takes into account the two sources of VA information. The performance of the hybrid integration scheme is compared to that of conventional integration schemes using simple FR quality measures, and we find that the proposed method offers greater performance improvement than the previous approaches.

Here, before the details of our method is presented, we briefly review the above-mentioned simple and popularly used FR image quality measures. As was done in [3] and [5], we have used the peak signal-to-noise ratio (PSNR) and structural similarity (SSIM) [8] in this paper to compare the performance of different VA integration policies. The PSNR is computed as follows:

$$
\begin{aligned}
& \text { PSNR }=20 \log _{10}\left(\frac{255}{\mathrm{MSE}}\right), \\
& \text { where MSE }=\frac{1}{N} \sum_{i=1}^{N}\left(x_{i}-y_{i}\right)^{2} .
\end{aligned}
$$

In (1), $x_{i}$ and $y_{i}$ denote the $i$ th pixel in the original image $\mathbf{x}$ and the test image $\mathbf{y}$, respectively, and $N$ denotes the total number of pixels in the image. The SSIM [8] basically measures the similarity between the two images based on local comparison of luminance, contrast, and structure.

\section{Proposed Hybrid Integration Approach}

As demonstrated in the previous studies [3], [5], both taskfree and quality-task saliency maps are beneficial to the evaluation of image quality, to a greater or lesser extent. In this paper, we exploit the fact that each map is unique and informative in a different way (see examples in Fig. 1 (d) and (f)). The system diagram of the proposed method is illustrated in 


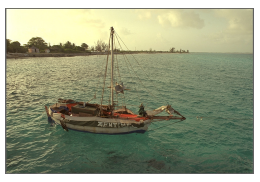

(a)

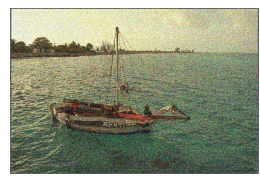

(b)

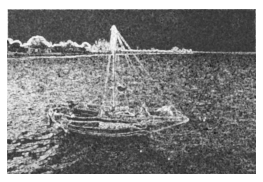

(c)

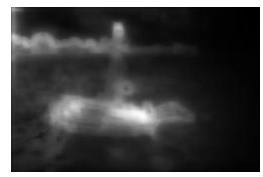

(d)

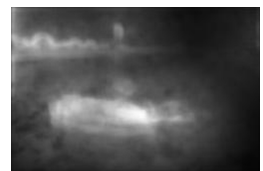

(f)

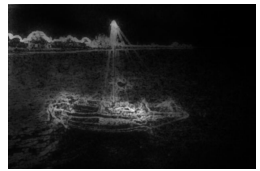

(e)

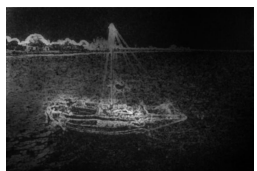

(g)
Fig. 1 Example of a quality metric building upon saliency. (a) Original image. (b) Test image (degraded by white Gaussian noise). (c) Distortion map of SSIM [8]. (d) Task-free saliency map. (e) Distortion map weighted by task-free saliency in (d). (f) Quality-task saliency map. (g) Distortion map weighted by quality-task saliency in (f). Note that, in this example, Goferman et al.'s algorithm [9] is adopted to generate saliency maps. In the saliency maps, the larger the pixel intensity is, the more likely the pixel attracts the observer's attention.

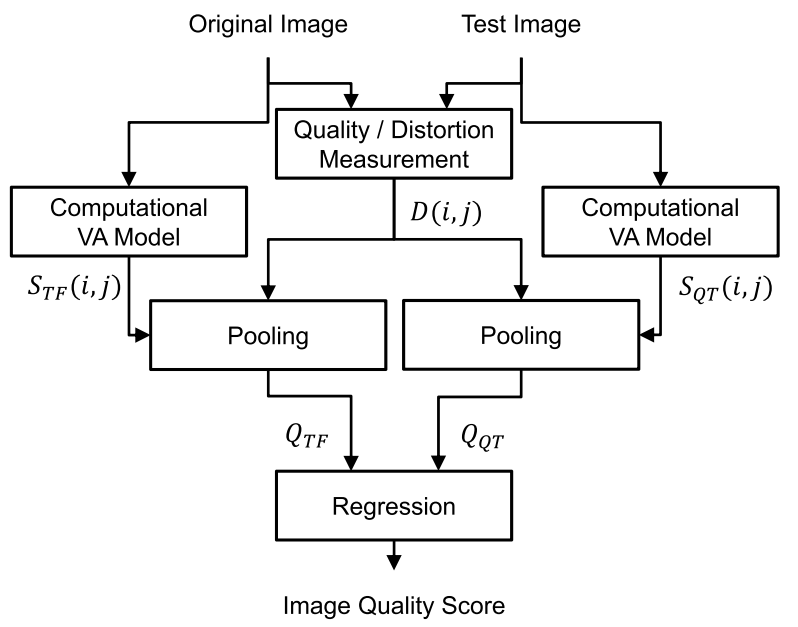

Fig. 2 System diagram of the proposed method.

Fig. 2. In each pooling stage, the saliency map is used as a weighting function as follows (see examples in Fig. 1 (e) and $(\mathrm{g}))$ :

$$
\begin{aligned}
\mathrm{Q}_{\mathrm{TF}} & =\frac{\sum_{i, j} \mathrm{~S}_{\mathrm{TF}}(i, j) \mathrm{D}(i, j)}{\sum_{i, j} \mathrm{~S}_{\mathrm{TF}}(i, j)}, \\
\mathrm{Q}_{\mathrm{QT}} & =\frac{\sum_{i, j} \mathrm{~S}_{\mathrm{QT}}(i, j) \mathrm{D}(i, j)}{\sum_{i, j} \mathrm{~S}_{\mathrm{QT}}(i, j)},
\end{aligned}
$$

where $\mathrm{S}_{\mathrm{TF}}$ and $\mathrm{S}_{\mathrm{QT}}$ denote the task-free and quality-task saliency maps, respectively, and D denotes the distortion map. In the existing VA-based approaches, $\mathrm{Q}_{\mathrm{TF}}$ or $\mathrm{Q}_{\mathrm{QT}}$ is directly used as a final image quality score. Differently from them, we obtain a final quality score by combining both $\mathrm{Q}_{\mathrm{TF}}$ and $\mathrm{Q}_{\mathrm{QT}}$. To that end, in this paper, we perform regression
Table 1 PLCC and SROCC of different VA integration approaches. TID2008 database [12]

\begin{tabular}{|l|c|c|c|c|}
\hline & PLCC & Gain (\%) & SROCC & Gain (\%) \\
\hline PSNR & 0.5190 & - & 0.5531 & - \\
\hline $\begin{array}{l}\text { PSNR-TF } \\
\text { [2], [3], [5]-[7] }\end{array}$ & 0.5381 & 3.7 & 0.5448 & -1.5 \\
\hline PSNR-QT [3], [5] & 0.5314 & 2.4 & 0.5428 & -1.8 \\
\hline PSNR-HYBRID & $\mathbf{0 . 6 4 7 2}$ & $\mathbf{2 4 . 7}$ & $\mathbf{0 . 6 4 1 7}$ & $\mathbf{1 6 . 0}$ \\
\hline \hline & PLCC & Gain (\%) & SROCC & Gain (\%) \\
\hline SSIM & 0.6012 & - & 0.6251 & - \\
\hline SSIM-TF & 0.6964 & 15.8 & 0.6956 & 11.3 \\
[2], [3], [5]-[7] & & & & \\
\hline SSIM-QT [3], [5] & 0.6845 & 13.9 & 0.7051 & 12.8 \\
\hline SSIM-HYBRID & $\mathbf{0 . 7 3 5 4}$ & $\mathbf{2 2 . 3}$ & $\mathbf{0 . 7 2 0 6}$ & $\mathbf{1 5 . 3}$ \\
\hline
\end{tabular}

Note that, in this table, the Goferman's VA model [9] is used.

as shown in Fig. 2. Note that the input to the regression is a two-dimensional vector $\left[\mathrm{Q}_{\mathrm{TF}} \mathrm{Q}_{\mathrm{QT}}\right]$, and $\mathrm{MOS}$ (mean opinion score) or DMOS (differential mean opinion score) is used as a regression target. We employed the support vector regression (SVR) with the radial basis function kernel in our work as was done in previous studies [10], [11]. In the SVR, the following function is learned:

$$
f(\mathbf{a})=\mathbf{w}^{\top} \varphi(\mathbf{a})+\gamma
$$

where $\varphi(\mathbf{a})$ is a nonlinear function of feature vector $\mathbf{a}(=$ $\left[\mathrm{Q}_{\mathrm{TF}} \mathrm{Q}_{\mathrm{QT}}\right]$ ), and $\mathbf{w}$ and $\gamma$ denote the weight vector and the bias term, respectively. In the training phase, the unknowns $\mathbf{w}$ and $\gamma$ are estimated to obtain the desired function in (4).

\section{Experimental Results}

In our experiments, we have used two publicly accessible subject-rated image databases: TID2008 database [12] and LIVE database [13]. We have employed two evaluation criteria: Pearson linear correlation coefficient (PLCC) and Spearman rank order correlation coefficient (SROCC). To demonstrate the effectiveness of the proposed method, we have used the cross validation scheme: we have randomly split the set of images into 25 subsets (i.e., 25-fold cross validation). In each fold, 24 subsets are used for training and the remaining subset is used for testing: in the testing phase, training examples are not included. In this paper, to show the generality of our approach, we have used two different computational VA models: Goferman's algorithm [9] and Bruce's algorithm [14]. Note that, as was done in [3], we have not conducted any nonlinear fitting to better visualize differences in performance: we have directly used the PLCC and SROCC between the final quality score (obtained from the algorithm) and the MOS or DMOS. We first report the performance of different VA integration approaches on the TID2008 database [12] in Tables 1 and 2. In the tables, "TF" and "QT" indicate task-free saliency map-based (in (2)) and quality-task saliency map-based (in (3)) integrations, respectively, and "HYBRID" indicates our integration approach. Note that the Goferman's VA model [9] and Bruce's VA model [14] are used in Tables 1 and 2, respectively. As shown in the tables, "performance gains" with the proposed 
Table 2 PLCC and SROCC of different VA integration approaches. TID2008 database [12]

\begin{tabular}{|l|c|c|c|c|}
\hline & PLCC & Gain (\%) & SROCC & Gain (\%) \\
\hline PSNR & 0.5190 & - & 0.5531 & - \\
\hline $\begin{array}{l}\text { PSNR-TF } \\
\text { [2], [3], [5]-[7] }\end{array}$ & 0.5339 & 2.9 & 0.5452 & -1.4 \\
\hline PSNR-QT [3], [5] & 0.5311 & 2.3 & 0.5424 & -1.9 \\
\hline PSNR-HYBRID & $\mathbf{0 . 6 4 7 9}$ & $\mathbf{2 4 . 8}$ & $\mathbf{0 . 6 4 6 1}$ & $\mathbf{1 6 . 8}$ \\
\hline \hline & PLCC & Gain (\%) & SROCC & Gain (\%) \\
\hline SSIM & 0.6012 & - & 0.6251 & - \\
\hline SSIM-TF & 0.6863 & 14.2 & 0.6806 & 8.9 \\
[2], [3], [5]-[7] & & & & \\
\hline SSIM-QT [3], [5] & 0.6756 & 12.4 & 0.6686 & 7.0 \\
\hline SSIM-HYBRID & $\mathbf{0 . 7 1 7 1}$ & $\mathbf{1 9 . 3}$ & $\mathbf{0 . 6 9 2 7}$ & $\mathbf{1 0 . 8}$ \\
\hline
\end{tabular}

Note that, in this table, the Bruce's VA model [14] is used.

Table 3 PLCC and SROCC of different VA integration approaches and PLCC for individual distortion types.

LIVE database [13]

\begin{tabular}{|l|c|c|c|c|}
\hline & PLCC & Gain (\%) & SROCC & Gain (\%) \\
\hline PSNR & 0.8252 & - & 0.8756 & - \\
\hline $\begin{array}{l}\text { PSNR-TF } \\
\text { [2], [3], [5]-[7] }\end{array}$ & 0.8734 & 5.8 & 0.8943 & 2.1 \\
\hline PSNR-QT [3], [5] & 0.8692 & 5.3 & 0.8899 & 1.6 \\
\hline PSNR-HYBRID & $\mathbf{0 . 9 1 4 6}$ & $\mathbf{1 0 . 8}$ & $\mathbf{0 . 9 3 2 6}$ & $\mathbf{6 . 5}$ \\
\hline \hline & PLCC & Gain (\%) & SROCC & Gain (\%) \\
\hline SSIM & 0.8585 & - & 0.9104 & - \\
\hline SSIM-TF & 0.8881 & 3.4 & 0.9416 & 3.4 \\
[2], [3], [5]-[7] & & & & \\
\hline SSIM-QT [3], [5] & 0.8851 & 3.1 & 0.9410 & 3.4 \\
\hline SSIM-HYBRID & $\mathbf{0 . 9 2 4 4}$ & $\mathbf{7 . 7}$ & $\mathbf{0 . 9 5 1 4}$ & $\mathbf{4 . 5}$ \\
\hline
\end{tabular}

\begin{tabular}{|l|c|c|c|c|c|}
\hline & JP2K & JPEG & WN & GB & FF \\
\hline PSNR & 0.8747 & 0.8650 & 0.9792 & 0.7744 & 0.8753 \\
\hline $\begin{array}{l}\text { PSNR-TF } \\
\text { [2], [3], [5]-[7] }\end{array}$ & 0.8926 & 0.8798 & 0.9793 & 0.8040 & 0.8940 \\
\hline PSNR-QT [3], [5] & 0.8924 & 0.8751 & 0.9791 & 0.7946 & 0.8908 \\
\hline PSNR-HYBRID & $\mathbf{0 . 9 1 8 8}$ & $\mathbf{0 . 8 9 5 4}$ & $\mathbf{0 . 9 7 9 8}$ & $\mathbf{0 . 8 1 6 1}$ & $\mathbf{0 . 9 1 6 7}$ \\
\hline \hline & JP2K & JPEG & WN & GB & FF \\
\hline SSIM & 0.9062 & 0.9245 & 0.9645 & 0.8494 & 0.9010 \\
\hline $\begin{array}{l}\text { SSIM-TF } \\
\text { [2], [3], [5]-[7] }\end{array}$ & 0.9439 & 0.9682 & 0.9716 & 0.9305 & 0.9361 \\
\hline SSIM-QT [3], [5] & 0.9421 & 0.9636 & 0.9730 & 0.9129 & 0.9336 \\
\hline SSIM-HYBRID & $\mathbf{0 . 9 6 1 3}$ & $\mathbf{0 . 9 7 7 8}$ & $\mathbf{0 . 9 7 6 8}$ & $\mathbf{0 . 9 4 7 9}$ & $\mathbf{0 . 9 5 5 4}$ \\
\hline
\end{tabular}

Note that, in this table, the Bruce's VA model [14] is used.

hybrid approach are higher than those with the task-free saliency map-based and quality-task saliency map-based integration approaches. We further demonstrated the superiority of our method on the LIVE database [13] in Table 3. In addition, in Table 3, we examined the effects of the proposed method on different image distortion types: JPEG2000 compression (JP2K), JPEG compression (JPEG), white noise (WN), Gaussian blur (GB), and fast fading (FF). From the table, we can see that our proposed approach leads to consistent improvement for different image distortions.

\section{Conclusion}

In this paper, we have presented a hybrid VA integration approach which takes advantages of task-free and quality-task saliency maps. The proposed method has simultaneously incorporated responses (i.e., VA during free viewing of unimpaired images and that during image quality assessment) of the human visual system into image quality measures. The performance of our proposed scheme was compared to that of conventional schemes using simple FR quality measures. Our results show that the proposed VA integration method performs better than the existing VA integration techniques.

\section{References}

[1] Z. Wang, "Applications of objective image quality assessment methods," IEEE Signal Process. Mag., vol.28, no.6, pp.137-142, 2011.

[2] A.K. Moorthy and A.C. Bovik, "Visual importance pooling for image quality assessment," IEEE J. Sel. Top. Signal Process., vol.3, no.2, pp.193-201, 2009.

[3] H. Liu and I. Heynderickx, "Visual attention in objective image quality assessment: Based on eye-tracking data," IEEE Trans. Circuits Syst. Video Technol., vol.21, no.7, pp.971-982, 2011.

[4] U. Engelke, H. Kaprykowsky, H.-J. Zepernick, and P. Ndjiki-Nya, "Visual attention in quality assessment," IEEE Signal Process. Mag., vol.28, no.6, pp.50-59, 2011.

[5] M.C.Q. Farias and W.Y.L. Akamine, "On performance of image quality metrics enhanced with visual attention computational models," Electron. Lett., vol.48, no.11, pp.631-633, 2012.

[6] I. Gkioulekas, G. Evangelopoulos, and P. Maragos, "Spatial Bayesian surprise for image saliency and quality assessment," Proc. IEEE International Conference on Image Processing, pp.1081-1084, 2010.

[7] A. Guo, D. Zhao, S. Liu, X. Fan, and W. Gao, "Visual attention based image quality assessment," Proc. IEEE International Conference on Image Processing, pp.3297-3300, 2011.

[8] Z. Wang, A.C. Bovik, H.R. Sheikh, and E.P. Simoncelli, "Image quality assessment: From error visibility to structural similarity," IEEE Trans. Image Process., vol.13, no.4, pp.600-612, 2004.

[9] S. Goferman, L. Zelnik-Manor, and A. Tal, "Context-aware saliency detection," IEEE Trans. Pattern Anal. Mach. Intell., vol.34, no.10, pp.1915-1926, 2012.

[10] M. Narwaria and W. Lin, "Objective image quality assessment based on support vector regression," IEEE Trans. Neural Netw., vol.21, no.3, pp.515-519, 2010.

[11] M. Narwaria and W. Lin, "Scalable image quality assessment with 2D mel-cepstrum and machine learning approach," Pattern Recognit., vol.45, no.1, pp.299-313, 2012.

[12] http://www.ponomarenko.info/tid2008.htm

[13] http://live.ece.utexas.edu/research/quality/

[14] N.D.B. Bruce and J.K. Tsotsos, "Saliency, attention, and visual search: An information theoretic approach," J. Vision, vol.9, no.3, pp.1-24, 2009. 